Proc. Indian Acad. Sci. (Chem. Sci.), Vol. 98, Nos 5 \& 6, June 1987, pp. 391-407.

(C) Printed in India.

\title{
Photoreactions in hydrophobic pockets
}

\author{
S DEVANATHAN, M S SYAMALA and V RAMAMURTHY*+ \\ Department of Organic Chemistry, Indian Institute of Science, Bangalore 560012 , India \\ +Present Address: E 328/208, CR \& D Experimental station, E I, Du Pont, Wilmington, \\ Del 19898, USA
}

\begin{abstract}
Organic chemists have long recognized the important role that reaction media play in controlling rates, product distributions and stereochemistry. Recently, much effort has been directed towards the use of organized media to modify reactivity as compared to that in isotropic liquids. Judicious selection of a given organized system for a given application requires sufficient understanding of the properties of the organized media themselves and those of the substrate interactions therein. The multimolecular aggregation of hydrophobic solutes in water could prove to be of immense value to the organic chemist. The aggregation of simple olefinic systems in water would enable photocycloaddition to compete efficiently with the various other modes of dacay of the short-lived excited state. Investigations of a few systems (dimerization of coumarins, stilbenes and alkylcinnamates) in our laboratory have been successful and they bring to light the significance of the hydrophobic effect. One of the most accepted manifestations of the hydrophobic interactions is probably the formation of micellar aggregates in aqueous solutions. Micelles provide a unique interface between aqueous and non-aqueous phases at which the non-polar solute can orient itself. While intermolecular orientation at micellar interfaces can provide selectivity in dimerization reactions, intramolecular orientation can be utilized to bring about selectivity in unimolecular photo-transformations. Such examples are presented.
\end{abstract}

Keywords. Photoreactions; association in aqueous media; micellar effect; selectivity in reactions; hydrophobic effect.

\section{Introduction}

Hydrophobic interactions are often claimed to play a central role in most biological processes. It is described as the indirect contributor to the intermolecular forces arising due to the surrounding aqueous or mixed aqueous solvent (Ben Naim 1980). The so-called 'direct' forces are those which operate in the gas as well as in solution phases, namely van der Waals' interactions, London forces and Columbic forces. Interestingly, the non-polar solutes present in the aqueous phase experience, in addition to the 'direct' forces, yet another intermolecular force termed as the hydrophobic force, solely due to the surrounding aqueous medium, tending to cluster them together. Traditionally, the hydrophobic effect has been ascribed to the relatively unfavourable entropy of dissolution of non-polar solutes in water in comparison with non-polar solvents, as a result of the formation of ordered 'ice berg' regions of water surrounding such solutes (Frank and Evans 1945). An interesting consequence of this is reflected in the tendency of apolar solutes to

*To whom all correspondence should be addressed. 
aggregate in the aqueous phase, thus minimizing the loss of entropy. Although the hydrophobic effect has been viewed as an entropy effect in the past, recent reports portray enthalpy as the overriding contributor (Abraham 1982; Wertz 1980). The multimolecular aggregation of hydrophobic solutes in water could prove to be of immense value to the organic chemist. Probably, the appreciation of hydrophobic interactions began with the discovery of micelle formation, and their introduction as a reaction medium, an area which has stimulated considerable interest among chemists (Ramnath et al 1985; Ramamurthy 1986). More recently, however, the uniqueness of the aqueous phase as a reaction medium was demonstrated (Rideout and Breslow 1980). Although the utility of the aqueous phase as a reaction medium for thermal reactions could be appreciated, at the time we began our studies its use for photoreactions was yet to be demonstrated. With this aim in mind, we undertook an estimation of the potential of aqueous and micellar phases as media for photoreactions. In the present article the results of our investigations are described with the aim of illustrating and highlighting the significant role of hydrophobic interactions in altering the course of photoreactions in aqueous and micellar phases.

\section{Water: A unique and useful solvent}

The preassociation of simple organic solutes in aqueous media as a result of hydrophobic interactions can be utilised in bringing about efficient bimolecular reactions. The presence of microheterogeneous regions of high local concentrations of solutes as well as the formation of a transition state with a smaller surface area would serve as driving forces for such bimolecular reactions. A dramatic illustration of this phenomenon was made in the Diels-Alder reactions of cyclopentadiene with a variety of dienophiles in aqueous solutions (Rideout and Breslow 1980). Efficient cycloaddition occurred even when the reactants were poorly soluble and formed a separate phase in water (Breslow et al 1983). Moreover, a remarkable selectivity between the endo and exo cycloadducts was demonstrated (Breslow et al 1983; Grieco et al 1983). This was attributed to a superposition of the specific orientational effect along with the polarity effect of water. Prompted by the above results, efforts towards the utilisation of the preassociation of organic solutes in water in bringing about efficient and selective photodimerizations were thought to be in order. The aggregation of simple olefinic systems in water would enable photocycloaddition to compete efficiently with the various other modes of decay of the short-lived excited state. Thus, hydrophobic aggregation should lead to exciting prospects for the excited state chemists:

The effectiveness of pre-association in bringing about efficient photodimerization was originally demonstrated for concentrated aqueous solutions of dimethylthymine and tetramethyl uracil by Morrison and coworkers (Kleopfer and Morrison 1972; Otten et al 1977). Efficient photodimerization which resulted from the singlet excited state of these solutes was incompatible with a mechanism involving a diffusion controlled reaction between the short-lived singlets and the ground state molecules and hence required the presence of preformed aggregates in aqueous solution. The presence of such aggregates was detected by osmometric measurements and efficient photodimerization was attributed to the ground state stacking 
of these solutes in concentrated aqueous solution. In fact such a hydrophobic aggregation could also be expected in very dilute aqueous solutions of poorly soluble organic solutes and this could prove to be much more dramatic by bringing about photodimerization in very dilute solutions. Investigations of a few such systems in our laboratory have been successful and they bring to light the significance of the hydrophobic effect (S Devanathan and V Ramamurthy, unpublished results; Muthuramu and Ramamurthy 1982; Syamala and Ramamurthy 1986). Coumarin forms one such system with moderate solubility in water. The photodimerization of coumarin in non-aqueous media is known to be less efficient, yet regiospecific depending on the polarity of the solvent. While anti-head-head dimer was the major isomer in non-polar solvents, the syn-head-head was the major component in polar solvents $\left(\Phi_{\mathrm{dim}} \simeq 10^{-5}\right.$ at $0.02 \mathrm{M}$; scheme 1 ). Dramatically, a single product, namely, the syn-head-head dimer crystallised out when dilute aqueous solutions of coumarin were photolysed $\left(\Phi_{\text {dim }} \simeq 10^{-3}\right.$ at $\left.0.01 \mathrm{M}\right)$. Such an enhanced reactivity was unexpected based on polarity considerations alone. In line with the reported photodimerization of coumarin in non-aqueous solvents, the reaction was presumed to be singlet derived. In order for dimerization to compete efficiently with the other deactivating modes of the short-lived excited singlet state either the life:time of the singlet state should be longer in aqueous solution or reaction must occur from preformed aggregates. The failure to observe a deviation from Beer's law, which would support the aggregation proposal, does not completely rule out the possibility of ground state aggregation, as it is known from earlier investigations that ground state aggregation need not always be accompanied by a deviation in Beer's law. On the other hand, coumarin in aqueous solution was found to emit strongly in contrast to its poor emission in non-aqueous solvents, thus suggesting that the excited state lives long enough for emission to compete with other modes of deacay. Based on the above arguements it can be concluded that the enhanced efficiency of coumarin dimerization is a combined effect of its increased lifetime and the presence of hydrophobic aggregation in water.

Investigations of poorly water-soluble systems could lead to much more exciting results, and this was, in fact exemplified by the photodimerization of stilbene and alkyl cinnamates in dilute aqueous solution. Of the various reactions that originate

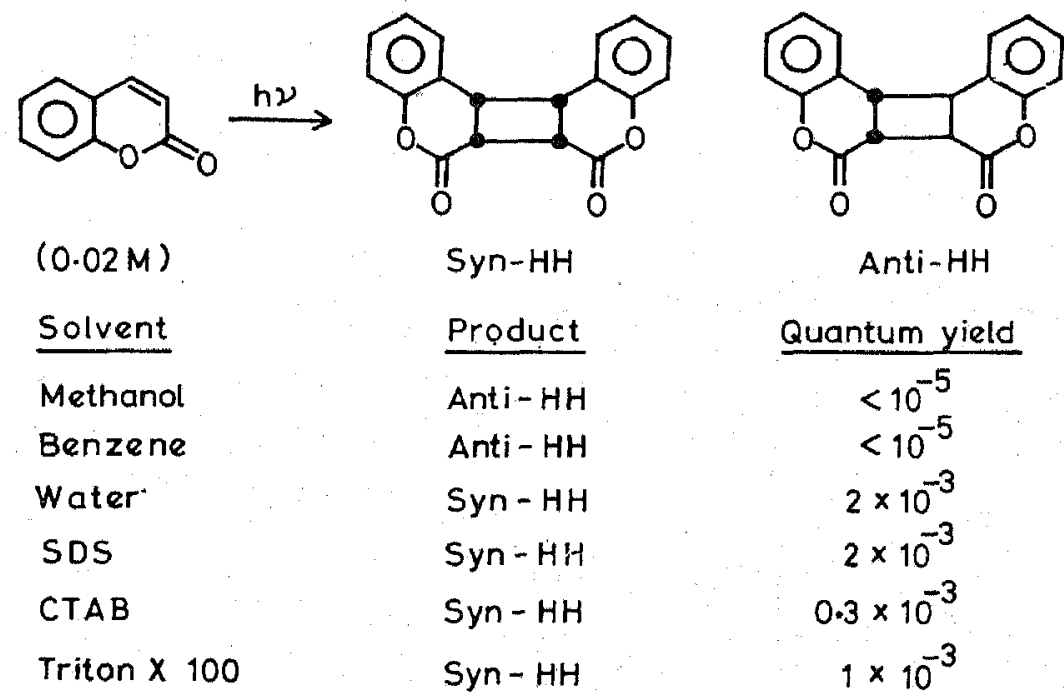

Scheme 1 


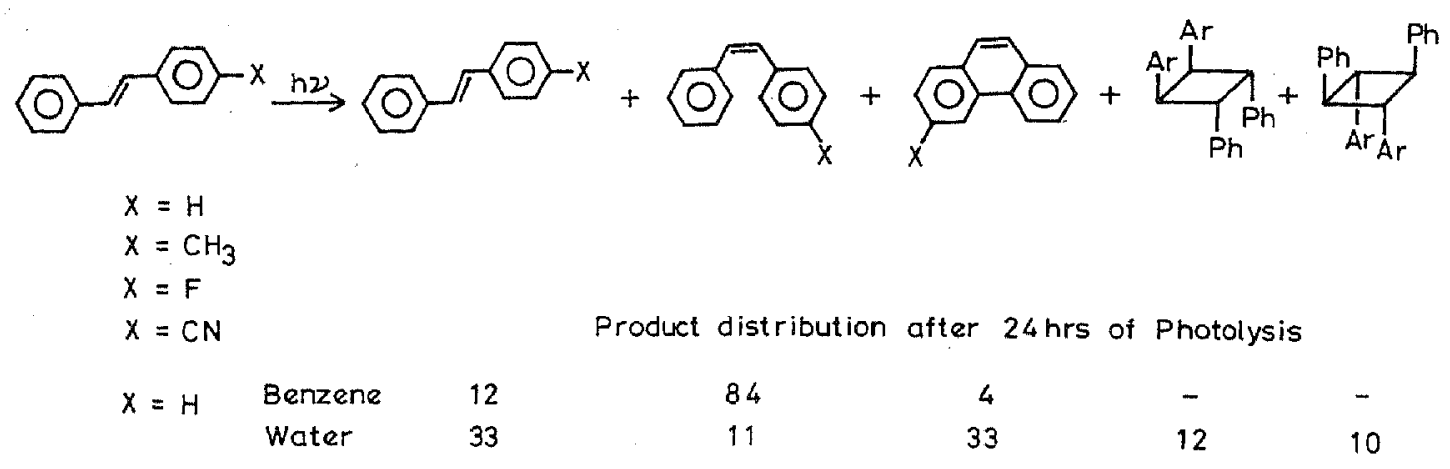

Scheme 2.

from their excited singlets, geometric isomerization and cyclization are the main competitors in dilute non-aqueous solutions of stilbene. Photodimerization is totally absent in organic solvents and begins to be important only above a concentration of $0.1 \mathrm{M}$. Surprisingly, a very dilute aqueous solution $\left(\sim 10^{-6} \mathrm{M}\right)$ of trans-stilbene underwent dimerization upon 24 hours of photolysis (scheme 2). In a similar manner, the formation of two isomeric dimers could be detected in a dilute aqueous solution $\left(=10^{-5} \mathrm{M}\right)$ of $c i s$-stilbene within 2 hours of photolysis. The two isomeric dimers detected by gas chromatographic analysis and $\mathrm{H}^{1}-\mathrm{NMR}$ proved to be the same as those formed in benzene irradiations, with the same ratio. The lack of stereoselectivity in aqueous media probably shows that the geometry of the excimer remains the same in aqueous and non-aqueous solvents. A follow-up of the product yield with respect to time revealed that isomerization preceded dimerization for cis-stilbene and the resulting trans iṣomer dimerized efficiently. The possible dimerization from floating micro-crystals of trans-stilbene was ruled out based on control irradiations of trans-stilbene crystals which proved to be photostable. As in the previous cases efficient dimerization of stilbene from their excited singlet state warrants ground state aggregation. In contrast to coumarin, were an increased lifetime (singlet) was proposed based on its high emission yield, the strongly fluorescent trans-stilbene underwent a noticeable drop in its emission yield in aqueous solution. This probably indicates the presence of additional decay channels, namely excimer formation and dimerization of the preformed aggregates, which deactivate the excited singlet state of stilbene.

Alkyl cinnamates behaved in a manner similar to the stilbene in that near quantitative dimerization occurred in dilute aqueous solution $\left(\sim 10^{-3} \mathrm{M}\right.$, scheme 3 ), while non-aqueous solutions of identical concentration led only to isomerization. The head-head dimer, $\delta$-truxinate was the major isomer detected by $\mathbf{H}^{1}$-NMR. The dimerization efficiency in aqueous solution was high in contrast to that of the neat liquid although the ratio of the isomeric dimers were identical in both the cases. The efficient dimerization in the aqueous phase reveals it to be a manifestation of the hydrophobic aggregation of solutes. To support the aggregation proposal in the case of stilbenes and cinnamates, experiments were devised with the use of additives expected to alter the aggregation behaviour in predictable ways. It is known that lithium chloride increases hydrophobic aggregation while guanidinium chloride serves to de-aggregate organic and biomolecules in water (von Hippel and Schleich 1969). Noticeable consequences of such perturbations were found in the resulting dimer yields (tables 1 and 2). In both the cases investigated, namely cis-stilbene and trans-ethyl cinnamate, lithium 


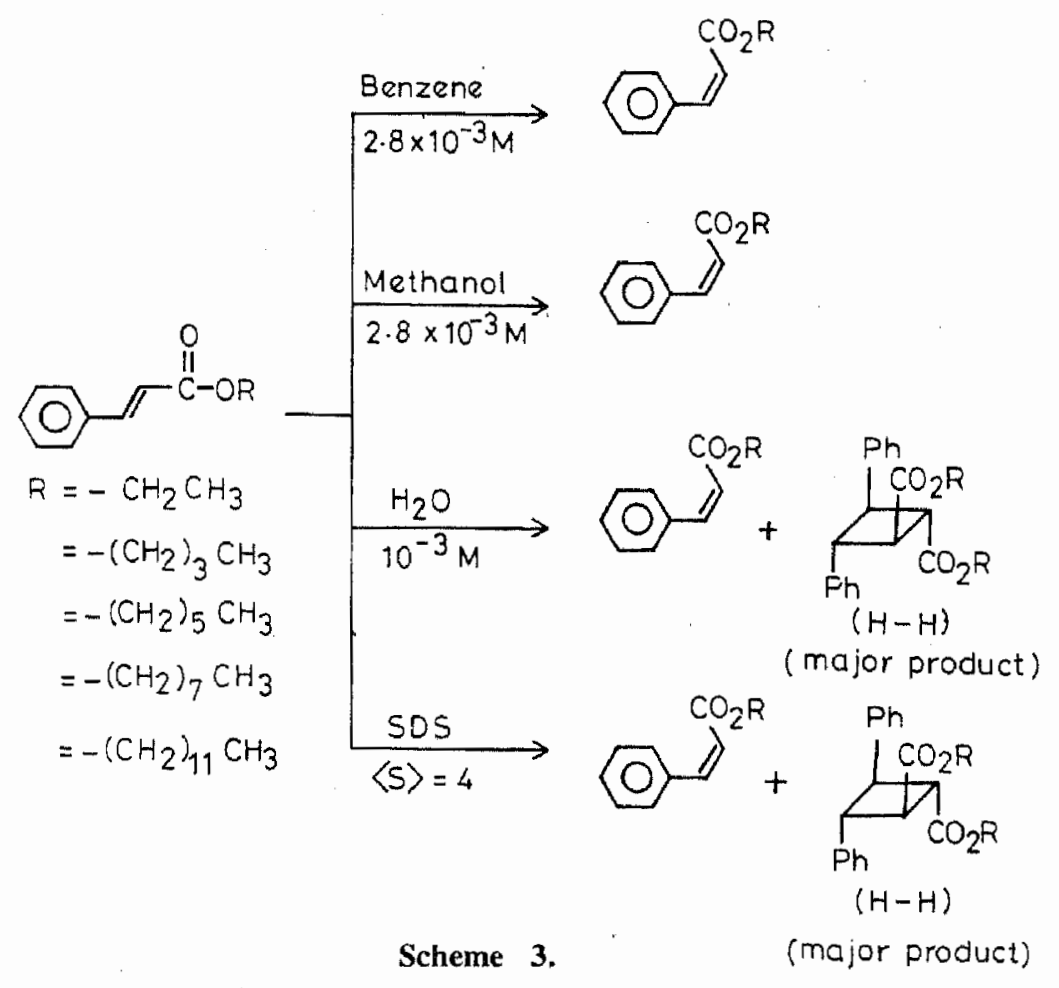

Table 1. Product distribution upon photolysis of stilbene in water in presence of additives $^{a, h}$.

\begin{tabular}{|c|c|c|c|c|c|}
\hline \multirow[b]{2}{*}{ Condition ${ }^{c}$} & \multicolumn{2}{|c|}{ Geometric isomers $(\%)$} & \multirow[b]{2}{*}{ Phenanthrene (\%) } & \multicolumn{2}{|c|}{ Dimer (\%) } \\
\hline & cis & trans & & $A$ & $B$ \\
\hline cis-stilbene in benzene & 91 & 5 & 4 & - & - \\
\hline cis-stilbene & 11 & 33 & 33 & 12 & 12 \\
\hline cis-stilbene + $\mathrm{LiCl}(3)$ & 7 & 27 & 25 & 25 & 17 \\
\hline $\begin{array}{l}\text { cis-stilbene + guani- } \\
\text { dinium chloride }(3 \mathrm{M})\end{array}$ & 11 & 21 & 53 & 8 & 6 \\
\hline $\begin{array}{l}\text { cis-stilbene }+\beta \text {-cyclo- } \\
\text { dextrin }(1: 10)\end{array}$ & 29 & 71 & - & - & - \\
\hline $\begin{array}{l}\text { cis-stilbene + SDS } \\
\quad\langle S\rangle: 1^{d}\end{array}$ & 36 & 26 & 33 & 3 & 2 \\
\hline
\end{tabular}

"Analysed by g.c.; Error limit: $\pm 2 \%$.

"Irradiated for $24 \mathrm{hr}$ using a $450 \mathrm{~W}$ medium pressure mercury lamp in a merry-go-round. "All solutions had identical concentration $\left(5.5 \times 10^{-4} \mathrm{M}\right)$ and matched $\mathrm{OD}$, and were irradiated simultaneously.

${ }^{d}\langle S\rangle=$ Occupancy number.

chloride enhanced the dimer yield while guanidinium chloride reduced it noticeably. In a similar manner, the encapsulation of these molecules into the hydrophobic cavity of $\beta$-cyclodextrin would isolate them from the other molecules thus preventing dimerization totally. This was indeed observed upon addition of an excess of $\beta$-cyclodextrin to aqueous solutions. Thus the presence of hydrophobic aggregation in dilute aqueous solution of stilbene and trans-cinnamates was proved to be the principal factor responsible for their efficient dimerization. The results 
Table 2. Photolysis of ethyl cinnamate in aqueous media in the presence of additives.

\begin{tabular}{|c|c|c|c|}
\hline \multirow{2}{*}{ Media $^{a, b}$} & \multicolumn{3}{|c|}{ Percentage of products ${ }^{c}$} \\
\hline & cis & trans & Dimer \\
\hline Water & 7 & 22 & 70 \\
\hline $\begin{array}{l}\text { Water/lithium } \\
\text { chloride }(3 \mathrm{M})\end{array}$ & 5 & 21 & 74 \\
\hline $\begin{array}{l}\text { Water/guanidinium } \\
\text { chloride }(3 \mathrm{M})\end{array}$ & 10 & 30 & 60 \\
\hline $\begin{array}{l}\text { Water/ } \beta \text {-cyclo- } \\
\text { dextrin }(1: 2)\end{array}$ & 56 & 43 & - \\
\hline $\begin{array}{l}\text { Water/ } \beta \text {-cyclo- } \\
\text { dextrin }(1: 5)\end{array}$ & 58 & 41 & - \\
\hline
\end{tabular}

"Solutions were of optical density.

${ }^{\prime}$ Irradiations were done using Rayonet reactor fitted with $R P R=254 \mathrm{~nm}$ lamps. All samples irradiated for $24 \mathrm{hr}$.

Based on GC analysis.

described above demonstrate the potential of commonly available water as a solvent for photoreactions and stress the need for an increased appreciation of its utility. Needless to say, hydrophobic interactions are the major contributions which make water a unique and useful solvent for photo and thermal reactions.

\section{Micelles as reaction vessels}

One of the most widely accepted manifestations of hydrophobic interactions is probably the formation of micellar aggregates in aqueous solution. Surfactant molecules with polar head groups and hydrophobic tails tend to cluster up in an aqueous medium forming an oil-like pool of hydrocarbon chains from which the polar head groups stick out and get solvated by water. Such spherical aggregates are called micelles and these form at and above a certain concentration range known as critical micellar concentration (CMC). The most interesting property of a micelle lies in its ability to solubilise non-polar solutes by providing a hydrophobic pocket for their residence in aqueous and non-aqueous phases at which the non-polar solute can orient itself suitably. The following section is devoted to an illustration of the potential of micelles as reaction vessels with the use of a few photoreactions investigated in our laboratory where striking alterations were brought about by the micellar media.

\subsection{Intermolecular orientation at micellar interface}

Apart from solubilizing apolar solutes, the micellar interface serves to orient them with their polar moities exposed to the aqueous exteriror, while the non-polar groups get buried in the interior. Such an orientation in fact organizes the micellesolubilized reactants in a predictable manner and is expected to lead to remarkable regioselectivities in micellar reactions. A dramatic illustration of this phenomenon which also proves to be of great synthetic utility is the regiospecific 
photodimerization of cyclopentenones in potassium dodecanoate (KDC) micelles, where exclusive formation of the head-head dimer was observed (Lee and de Mayo 1979; Berenjian et al 1982). This is in contrast to the dimerization behaviour in cyclohexane where the head-tail dimers predominate. Although an improved yield of the head-tail dimer could be brought about by increasing the polarity of the solvent used for irradiations, the total reversal of regioselectivity observed in KDC micelles is far more than expected based on polarity considerations alone and can only be attributed to the pre-orientation of the solute molecules at the miceliar surface.

While investigating the potential of micelles as reaction vessels in bringing about regioselective photodimerization reactions, two factors need to be borne in mind. Along with the pre-orienting effect at the micellar interface, polarity of the micelle would also play a role in deciding the regiochemical outcome of a reaction, especially for cases which are known to be sensitive to polarity effects. The situation is well-exemplified by the photodimerization of coumarin in micellar media (Muthuramu and Ramamurthy 1982, 1984). The reaction efficiency which was poor in organic solvents improved to a great extent in the various micelles investigated, presumably due to high local concentration of coumarin in the micelles. Interestingly, a single dimer (syn-head-head) crystallized out of the micellar solutions during irradiation. This was unexpected because the hydrocarbon-like interior of the micelle was likely to give the anti head-head dimer, the product of irradiation in non-polar solvents. The most obvious alternative, that of a reaction occurring in the aqueous phase with the micelle serving only as the storage site, was ruled out based on the insensitivity of the dimer yield to changes in polarity of the aqueous phase upon addition of sodium chloride and its sensitivity to an increase in the occupancy number of coumarin. Thus the regioselective dimerization could be accounted for by invoking a certain degree of water penetration into the micellar interior, and hence providing a polar environment for the coumarin molecule. It is to be noted that the required polarity for the reaction can be provided if the coumarin molecules align themselves at the micellar-water interface.

Although the regiochemical outcome of the above mentioned investigation was explainable based on a combination of the pre-orientational and polarity effects of the micelles, $a$, desire to estimate the relative efficiencies of each in bringing about regioselectivity in micellar reactions is irresistable. In this regard, we proceeded to estimate the effectiveness of pre-orientational effect in bringing about regioselectivities (Muthuramu et al 1983; Ramesh and Ramamurthy 1984a; Ramnath and Ramamurthy 1984). Quite predictably a few systems were chosen where the regiochemistry of photodimerization was independent of the polarity of the medium. During the course of our efforts we were led not only to appreciate the potential of the pre-orientational effect in bringing about regioselectivity, but also its limitations in the presence of other overriding factors.

The photodimerization of 2-substituted naphthalenes which, in principle, could lead to the cis and trans dimers was chosen for investigation as it was known to give polarity independent dimer yields (scheme 4). In non-aqueous solvents the trans dimer is the major product with the formation of trace amounts of products derived from the cis-dimer. Irradiation of 2-substituted naphathalenes $1-4$ in SDS, CTAB, CTAC and DTAC micelles gave rise to the ketones derived from the cis-dimers as 
the sole product. In fact a precipitation of the cis-dimer itself was observed in the case of 2-ethoxynaphthalene in CTAC micelles. In a similar manner, the substrates $\underline{5}$ and $\underline{6}$ gave rise to cage products derived from the cis dimer when irradiated in the same micelles (scheme 5). The enhanced dimerization efficiency was explainable by the local concentration effect. A pronounced increase in the dimerization efficiency in CTAC when compared to that in CTAB could be anticipated due to the well-known effect of $\mathrm{Br}^{-}$in bringing about an increased intersystem crossing, thus shortening the singlet lifetime, leading to a decreased reactivity (Ramesh and Ramamurthy 1984b). When the counterion is changed to $\mathrm{Cl}^{-}$, the heavy atom induced intersystem crossing is negligible and hence dimerization efficiency is

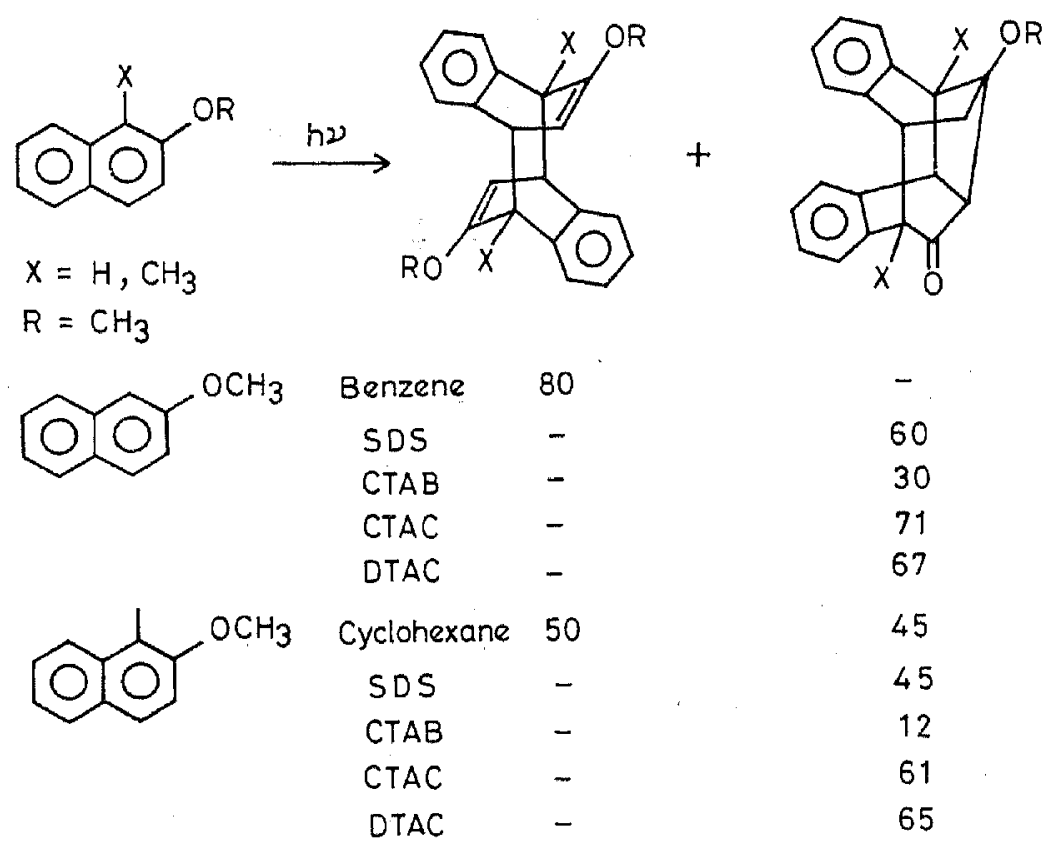

Scheme 4.

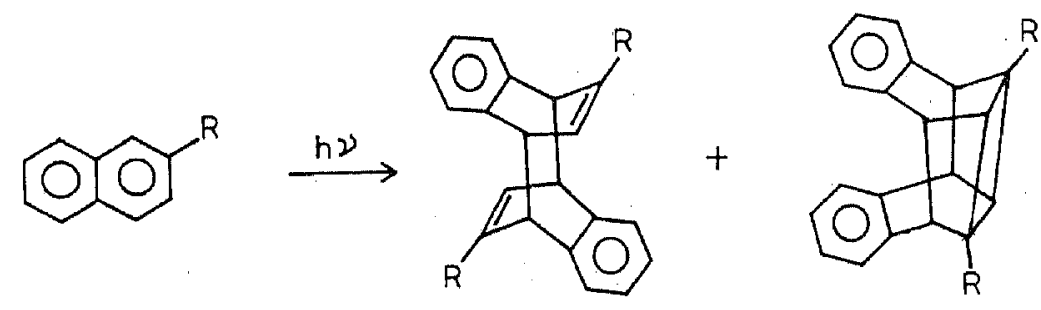

$\begin{array}{ll}\underline{5} & \mathrm{R}=\mathrm{COOCH}_{3} \\ \underline{6} & \mathrm{R}=\mathrm{CN}\end{array}$

$\underline{5}$

benzeneli-propanol

$25 \%$

SDS

$57 \%$

CTAC.

$36 \%$

6 benzene/hexane

$15 \%$

CTAC

$38 \%$

Scheme 5 . 
restored. The remarkable regioselectivity could only be explained based on the orientation of the 2-substituted naphthalenes at the micellar interface in a manner shown in figure 1, which is to be expected based on the amphiphilic nature of the solute. The present example thus serves as an illustration of the potential of pre-orientational effect in bringing about regioselectivity.

In order to examine the effectiveness of micelles in orienting molecules and to explore the possible limitations, we undertook the investigation of yet another system viz. 7-alkoxy and 4-methyl 7-alkoxy coumarins. These systems were appropriately chosen to suit our goal because these photodimerize in organic solvents to give rise to a single dimer ( $s y n$ head-tail) from their excited singlet states, irrespective of the polarity of the medium. It was anticipated that the introduction of a long alkyl chain at the alkoxy portion would help in anchoring the alkoxy chain in the interior of the micelle, while the carbonyl group would be turned towards the surface of the micelle. Such an orientation would facilitate the formation of the head-head dimer (figure 2). Our expectations of obtaining a head-head dimer from the irradiations of 7-alkoxy and 4-methyl 7-alkoxy coumarins in SDS, CTAB and CTAC micelles were in vain, the photolysis in all cases leading to the syn-head-tail dimer as the sole product, with a greater efficiency expected on the basis of the local concentration effect (table 3 ). The results thus demonstrate the insufficiency of the hydrophobicity introduced in terms of the alkyl chains to overcome the molecular forces to induce a reversal in the regioselectivity. We further attempted to bring about the expected reversal in selectivity by improving the degree of hydrophobicity, by introducing still longer chains. With these systems (7) also the formation of head-head dimer could not be achieved (scheme 6). Surprisingly, the substrate $\underline{7}$ with the longest alkyl chain,

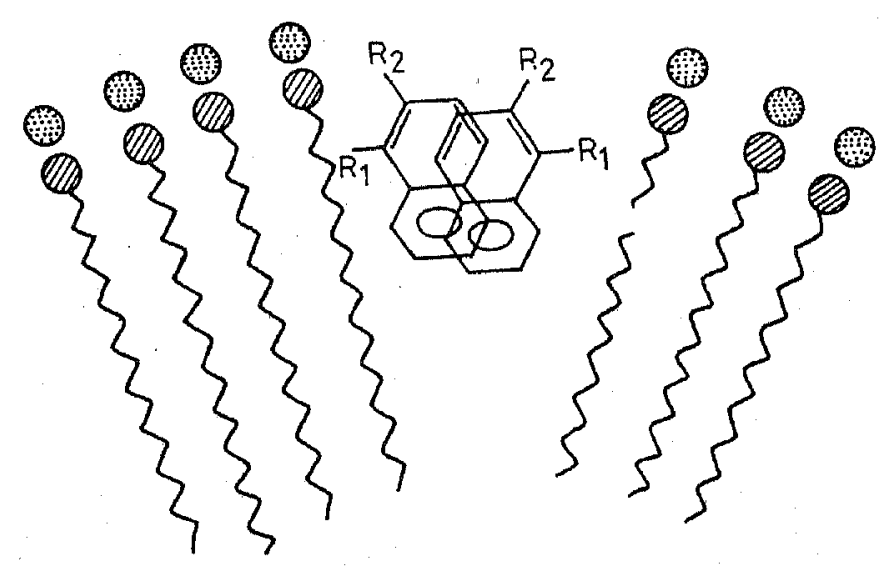

Head group

Counterion

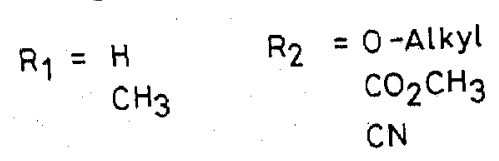

Figure 1. Orientation of 2-substituted naphthalenes at the micellar interface: 

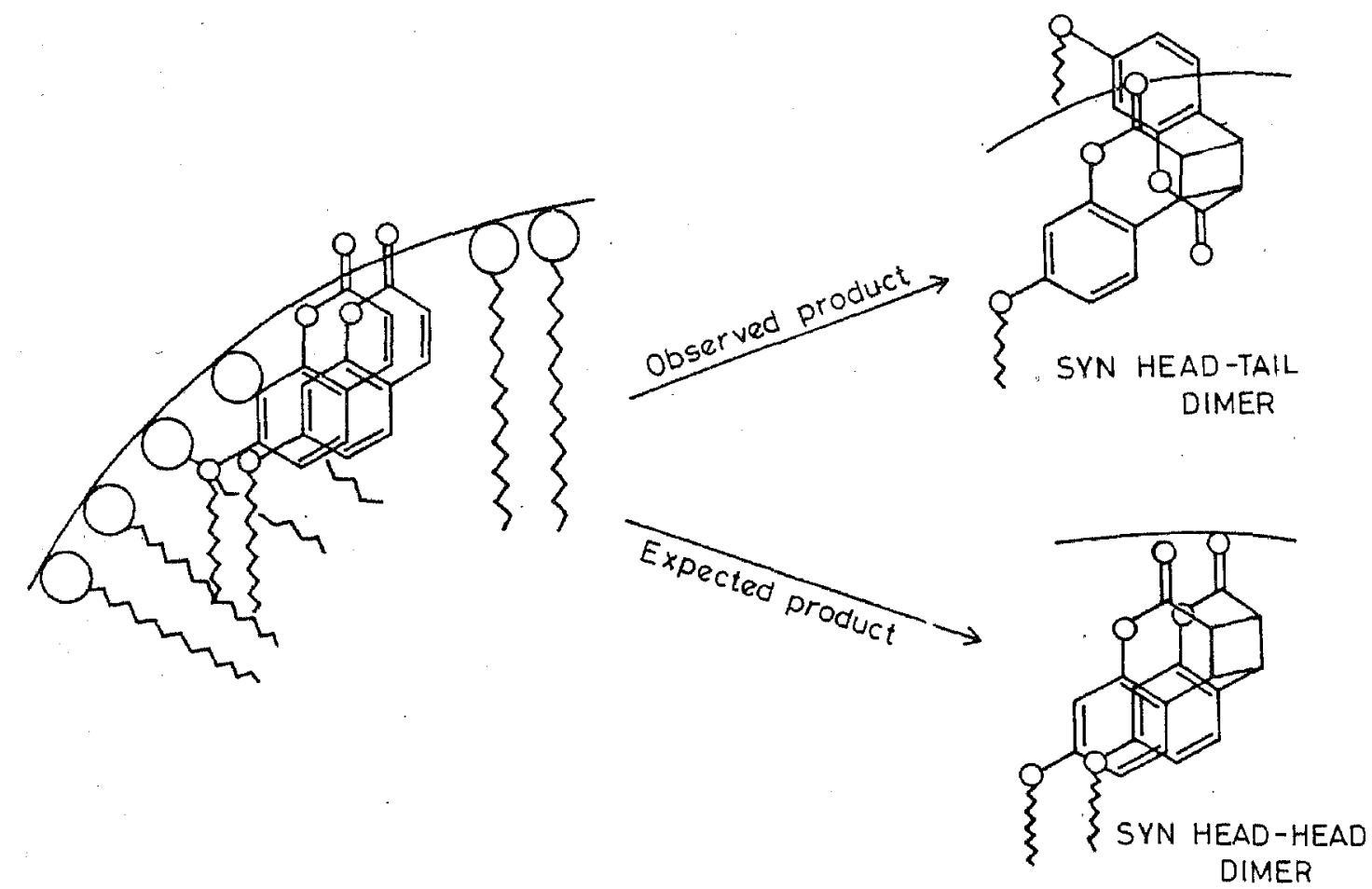

Figure 2. Expected orientation of long chain 7-alkoxycoumarins at the micellar interface.

which had a problem in getting solubilized into SDS micelles at high concentrations, upon photolysis gave rise to a mixture dominated by the anti-head-tail dimer. In fact, substrates with alkyl chains longer than the surfactant chains themselves are expected to be adsorbed on the surface of the micelle with the alkyl chain curled up inside the micelle. As a result of such an adsorption, two neighbouring molecules present on the surface could probably encounter each other only in a manner suitable for the formation of the anti-head-tail dimer (figure 3 ). In conclusion, the failure to obtain the expected head-head dimer in these systems by the induction of pre-orientation exposes the limitations of the micellar alignment effect when the molecular forces operating to dictate the regiochemical outcome are overridingly stronger than the hydrophobic interactions tending to orient the molecule at the micellar interface.

\subsection{Intramolecular orientation at micellar interface: Conformational and cage effects}

The hydrophobicity of the micellar interior which serves as the driving force for solubilization of apolar solutes, helps to prevent their escape from the interior of the micelle, by providing a more favourable environment for their existence, than the aqueous exterior. As a consequence of this, the short lived radicals formed in a' reaction are forced to stay in the same volume of the micelle long enough to encounter their counterparts. In other words, micelles act 'super cages' by trapping the radicals inside and facilitating their recombination. The 'cage effect' of micelles was utilized in a very imaginative manner in the photolysis of dibenzyl ketones (DBK) by Turro and co-workers (Turro et al 1980). Out of the three products AA, 
Table 3. Photodimsrization of 7-alkoxycoumarins in organic, aqueous and micellar media.

\begin{tabular}{|c|c|c|c|c|c|}
\hline Compound & Media & $\begin{array}{c}\text { (Coumarin) } \\
(\mathrm{M})\end{array}$ & $\begin{array}{l}\text { Duration of } \\
\text { irradiation } \\
\text { (hr) }\end{array}$ & $\begin{array}{l}\text { Product } \\
\text { formed }^{b}\end{array}$ & $\begin{array}{c}\text { Yield }^{c} \\
(\%)\end{array}$ \\
\hline $\begin{array}{l}\text { 7-O-Methyl- } \\
\text { coumarin (1) }\end{array}$ & $\begin{array}{l}\text { Methylene chloride } \\
\text { water } \\
\text { SDS }(0.032 \mathrm{M}) \\
\text { CTAB }(0.01 \mathrm{M}) \\
\text { methylene chloride/ } \\
\text { benzophenone }(0.1 \mathrm{M})\end{array}$ & $\begin{array}{l}.0 .25 \\
2.1 \times 10^{-3} \\
1.1 \times 10^{-3} \\
6.0 \times 10^{-3} \\
0.15\end{array}$ & $\begin{array}{r}72 \\
24 \\
5 \\
72 \\
72\end{array}$ & Syn HT & $\begin{array}{l}40 \\
65 \\
70 \\
10\end{array}$ \\
\hline $\begin{array}{l}\text { 7-O-butyl- } \\
\text { coumarin }(2)\end{array}$ & $\begin{array}{l}\text { Chloroform- } d \\
\text { water } \\
\text { SDS }(0.05 \mathrm{M}) \\
\text { CTAB }(0.02 \mathrm{M}) \\
\text { chloroform- } d / \text { benzo- } \\
\text { phenone }(0.2 \mathrm{M})\end{array}$ & $\begin{array}{l}0.5 \\
10^{-5} \\
1.4 \times 10^{-2} \\
1 \cdot 1 \times 10^{-2} \\
0.25\end{array}$ & $\begin{array}{l}30 \\
30 \\
30\end{array}$ & $\begin{array}{l}\text { Syn HT } \\
\text { No reaction } \\
\text { Syn HT } \\
\text { No reaction }\end{array}$ & $\begin{array}{r}50 \\
5\end{array}$ \\
\hline $\begin{array}{l}\text { 7-O-hexyl- } \\
\text { coumarin (3) }\end{array}$ & $\begin{array}{l}\text { Chloroform- } d \\
\text { water } \\
\text { SDS }(0.05 \mathrm{M}) \\
\text { CTAB }(0.025 \mathrm{M}) \\
\text { chloroform- } d / \text { benzo- } \\
\text { phenone }(0.2 \mathrm{M})\end{array}$ & $\begin{array}{l}0.75 \\
10^{-5} \\
1.4 \times 10^{-2} \\
1.6 \times 10^{-2} \\
0.25\end{array}$ & $\begin{array}{l}45 \\
45 \\
45 \\
45\end{array}$ & $\begin{array}{l}\text { Syn HT } \\
\text { No reaction } \\
\text { Syn HT } \\
\text { Syn HT } \\
\text { No reaction }\end{array}$ & $\begin{array}{r}42 \\
5\end{array}$ \\
\hline $\begin{array}{l}\text { 7-O-heptyl- } \\
\text { coumarin (4) }\end{array}$ & $\begin{array}{l}\text { Chloroform- } d \\
\text { water } \\
\text { SDS }(0 \cdot 05 \mathrm{M}) \\
\text { CTAB }(0.025 \mathrm{M}) \\
\text { chloroform- } d / \text { benzo- } \\
\text { phenone }(0.2 \mathrm{M})\end{array}$ & $\begin{array}{l}0.25 \\
\text { Not soluble } \\
6 \times 10^{-3} \\
6 \times 10^{-3} \\
0.25\end{array}$ & $\begin{array}{l}50 \\
50 \\
50 \\
50 \\
50\end{array}$ & $\begin{array}{l}\text { Syn HT } \\
\text { Syn HT } \\
\text { Syn HT } \\
\text { No reaction }\end{array}$ & $\begin{array}{r}50 \\
6\end{array}$ \\
\hline $\begin{array}{l}\text { 7-O-octyl- } \\
\text { coumarin (5) }\end{array}$ & $\begin{array}{l}\text { Chloroform-d } \\
\text { water } \\
\text { SDS }(0.05 \mathrm{M}) \\
\text { CTAB }(0.025 \mathrm{M}) \\
\text { chloroform- } d / \text { benzo- } \\
\text { phenone }(0.2 \mathrm{M})\end{array}$ & $\begin{array}{l}0.25 \\
\text { Not soluble } \\
6 \times 10^{-3} \\
6 \times 10^{-3} \\
0.25\end{array}$ & $\begin{array}{l}50 \\
50 \\
50 \\
50\end{array}$ & $\begin{array}{l}\text { Syn HT } \\
\text { Syn HT } \\
\text { Syn HT } \\
\text { No reaction }\end{array}$ & $\begin{array}{r}25 \\
5\end{array}$ \\
\hline $\begin{array}{l}\text { 7-O-dodecyl- } \\
\text { coumarin (6) }\end{array}$ & $\begin{array}{l}\text { Chloroform-d } \\
\text { water } \\
\text { SDS }(0.05 \mathrm{M}) \\
\text { CTAB }(0.025 \mathrm{M}) \\
\text { chloroform-d/benzo- } \\
\text { phenone }(0.2 \mathrm{M})\end{array}$ & $\begin{array}{l}0.25 \\
\text { Not soluble } \\
5 \times 10^{-3} \\
5 \times 10^{-3} \\
0.25\end{array}$ & $\begin{array}{l}50 \\
50 \\
50\end{array}$ & $\begin{array}{l}\text { Syn HT } \\
\text { Syn HT } \\
\text { Syn HT } \\
\text { No reaction }\end{array}$ & $\begin{array}{r}10 \\
5\end{array}$ \\
\hline
\end{tabular}

aAll irradiations were conducted in Pyrex vessels by using a $450 \mathrm{~W}$ medium pressure mercury lamp. ${ }^{b}$ Structures of the dimers were identified by their spectral properties.

c Yields presented correspond to TLC isolated yields.

$\mathrm{AB}$ and $\mathrm{BB}$ that are possible from the combination of the benzyl radicals $\mathrm{A}$ and $\mathrm{B}$, which result from the photolysis of the unsymmetrical $D B K(A-C O-B)$, the exclusive formation of $\mathrm{AB}$ was found in $\mathrm{HDTCl}$ micelles, demonstrating the efficiency of the super cage provided by the micelle. The cage effect, as expected, was proportional to the hydrophobic volume and hence to the surfactant chain 
<smiles>[R]c1ccc2ccc(=O)oc2c1</smiles>

7a<smiles>[R]c1ccc2c(C)cc(=O)oc2c1</smiles>

$\underline{7 b}$

$$
\begin{aligned}
\mathrm{R} & =\mathrm{O}\left(\mathrm{CH}_{2}\right)_{13}-\mathrm{CH}_{3} \\
& =O\left(\mathrm{CH}_{2}\right)_{15}-\mathrm{CH}_{3} \\
& =O\left(\mathrm{CH}_{2}\right)_{17}-\mathrm{CH}_{3}
\end{aligned}
$$

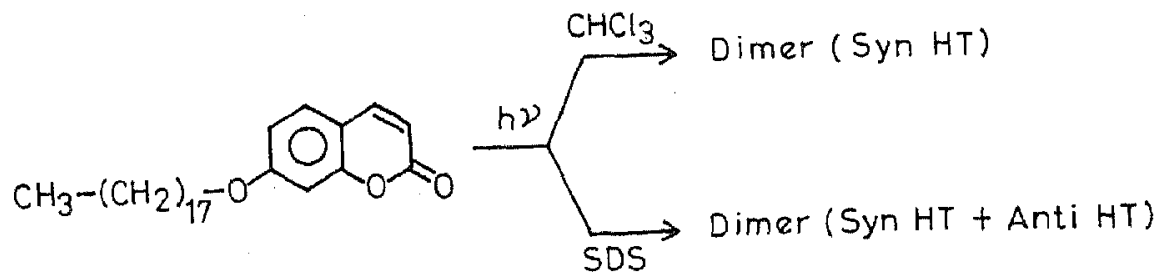

Scheme 6.

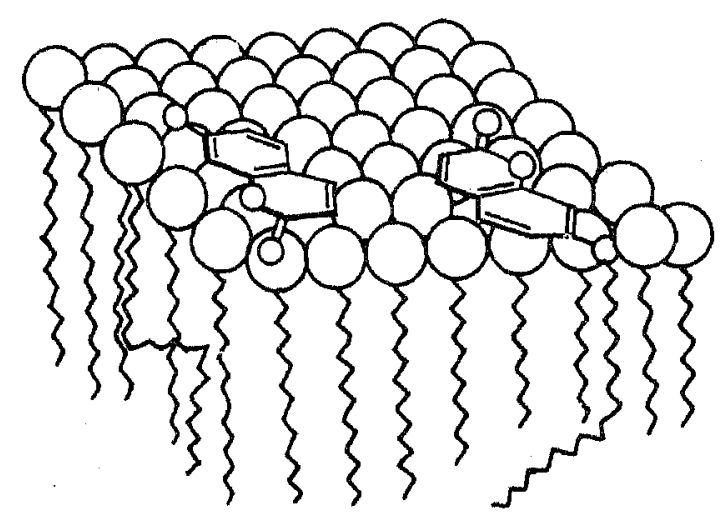

Figure 3. Pre-orientation of long chain 7-alkoxycoumarins at the micellar phase when the chain is longer than the surfactant alkylchain.

length. The cage control of a radical reaction could lead to very exciting prospects as was demonstrated by the achievement of an isotopic enrichment during the photolysis of DBK (Turro et al 1980). Another interesting consequence of the cage effect which hitherto has not received much attention was demonstrated in our laboratory in the photolysis benzoin alkyl ethers in a variety of micelles ( $S$ Devanathan and $\mathrm{V}$ Ramamurthy, unpublished results).

Benzoin alkyl ethers are known to undergo Norrish type I reaction as the only phtoreaction in organic solvents (scheme 7). The type II reaction, if feasible in these substrates, is not observed probably due to the overwhelmingly faster type I process. The incorporation of such a system into the super cage provided by the micelle, is expected to suppress the type I process, the initially formed $\alpha$-cleavage radical being facilitated to recombine to regenerate the starting benzoin ether. Under such circumstances, the normally slow type II process would become detectable, provided the conformation of the molecule is suitable for the 


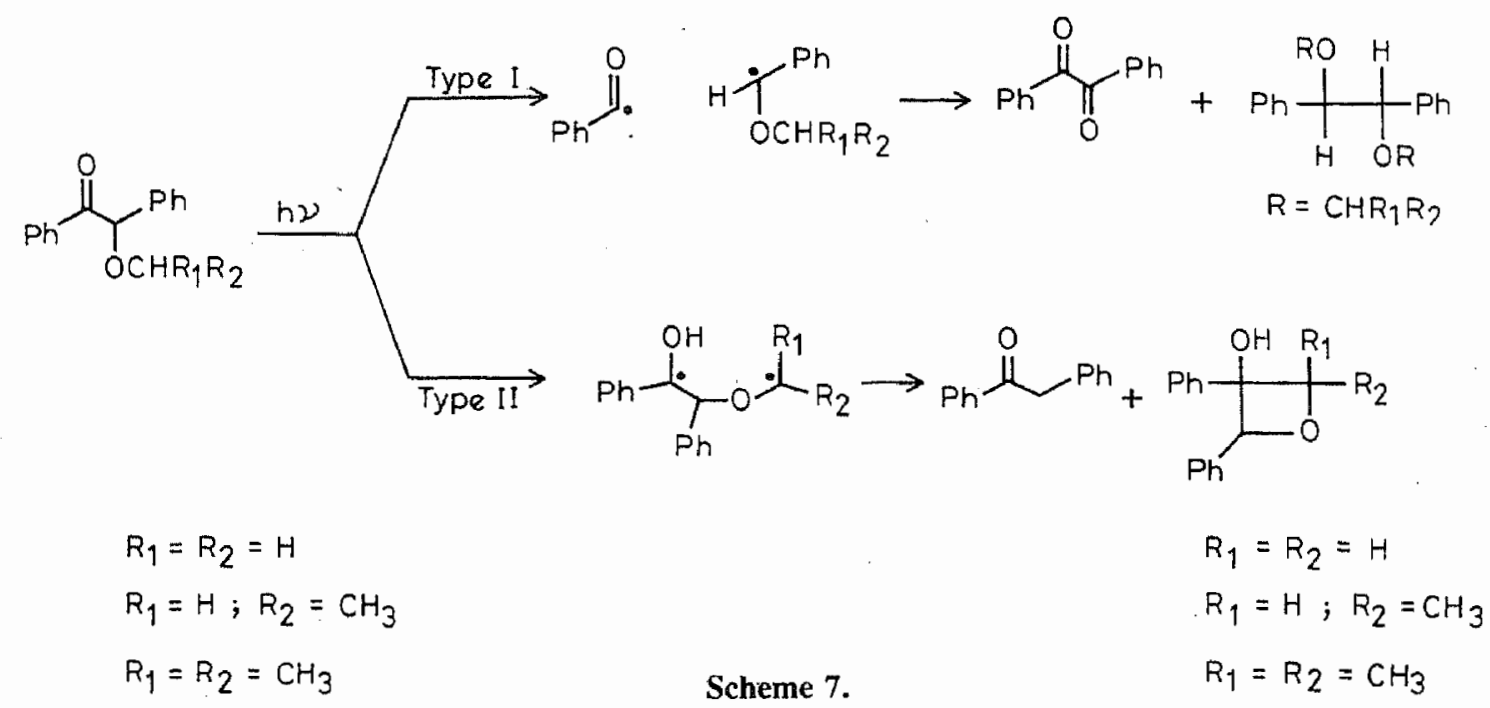

$\mathrm{H}$-abstraction process. In line with our expectations, photolysis of benzoin methyl ether in CTAB and CTAC micelles under conditions of low occupancy revealed the formation of the type II products along with the para-rearranged product of the starting benzoin ether in low yields (table 4). The super cage provided by the micelles probably allows the radicals to live long enough to tumble over before recombination, thus accounting for the small fraction of the rearranged product. The observed dependence of the type II products yeilds upon the occupancy number (table 5) and the hydrophobic volume (table 6) of the micelle gave support for the operation of cage control. It may be noted that the operatin of cage control alone does not suffice to explain the type II products. Out of the two conformations

Table 4. Photoproduct distribution of benzoinmethylether in various micelles.<smiles>COC(C(=O)Nc1ccccc1)C(c1ccccc1)(c1ccccc1)C(OC)c1ccccc1</smiles>

(Type I)

$\mathrm{CH}_{3}\left(\mathrm{CH}_{2}\right)_{11} \mathrm{SO}_{4}^{-} \mathrm{Na}^{+}$

(SDS)
$\mathrm{CH}_{3}\left(\mathrm{CH}_{2}\right)_{10} \mathrm{COO}^{-} \mathrm{K}^{+}$

(KDC)

DTAB

DTAC

$\mathrm{CH}_{3}\left(\mathrm{CH}_{2}\right)_{14} \mathrm{CH}_{2} \stackrel{+}{\mathrm{N}} \mathrm{H}_{4} \mathrm{Br}^{-}$

(CTAB)

23

42

38

44

50

$\mathrm{CH}_{3}\left(\mathrm{CH}_{2}\right)_{14} \mathrm{CH}_{2} \stackrel{+}{\mathrm{N}} \mathrm{H}_{4} \mathrm{Cl}^{-}$

(CTAC)
23<smiles>O=C(Cc1ccccc1)c1ccccc1</smiles><smiles>OC1(P)COC2OCC21c1ccccc1</smiles>
(RP)

\section{(Type II)}

12

10 
Table 5. Type I vs type II photoproduct distribution of benzoinmethylether in various media.

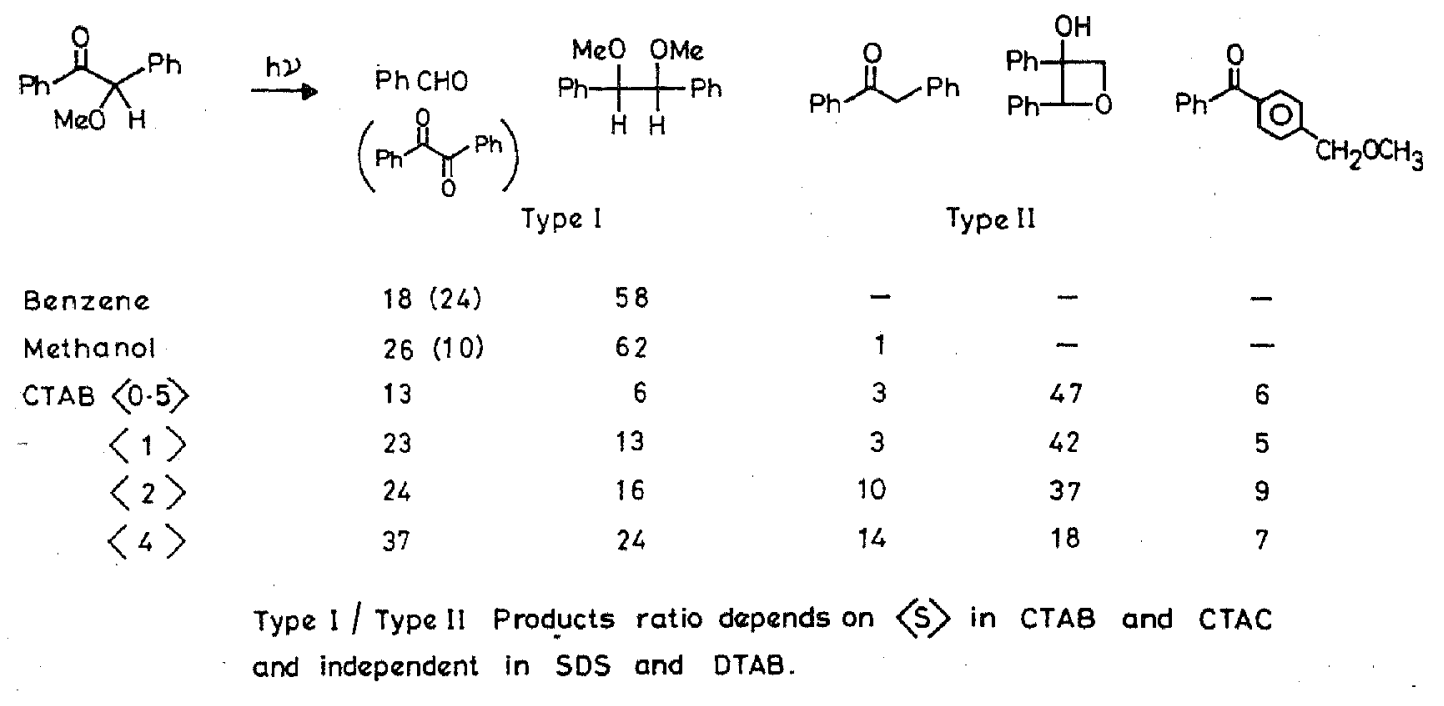

Table 6. Photoproduct distribution of benzoinoctylether in various micelles.

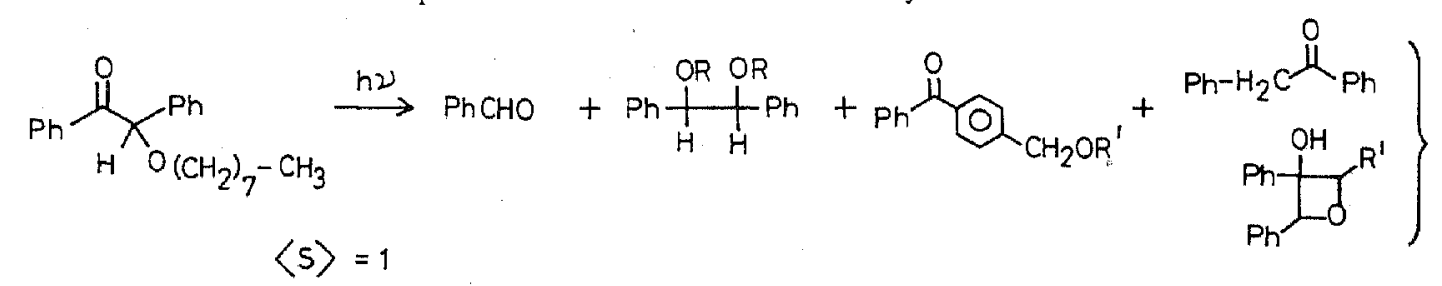

Micelle

$\begin{array}{rllll}\text { Sodium decyl sulphate } & 61 & 35 & - & <5 \\ \text { Sodium dodecyl sulphate } & 69 & 28 & - & <5 \\ \text { Potassium decanoate } & 62 & 36 & - & <5 \\ \text { Potassium dodecanoate } & 55 & 34 & - & <5 \\ \text { DTAB } & 64 & 32 & - & <5 \\ \text { DTAC } & 62 & 36 & - & <5 \\ \text { CTAB } & 28 & 15 & 47 & <5 \\ \text { CTAC } & 34 & 17 & 45 & <5\end{array}$

$A$ and $B$ shown in figure 4 for the benzoin alkyl ether, the specific orientation of a particular conformer B at the micellar interface (probably due to the need for the polar oxygens of the carbonyl and alkoxy groups to point towards the aqueous phase), would lead to the formation of the type II products, as this conformation is the one favourable for the $\mathrm{H}$-abstraction process. In order to investigate the validity of proposing such a conformational control in micelles, we went on to introduce longer alkyl chains on the alkoxy moiety, which would prefer to reside in the interior of the micellar core, thus attempting to populate conformer $\mathrm{A}$ to increasing extents. In such a case, even in the presence of a cage control, there is no possibility of observing the type II reaction. To our great delight photolysis of benzoin octyl ether in CTAB and CTAC micelles, gave rise only to the para-rearranged product (table 7) showing that the initially formed $\alpha$-cleavage radicals had the only choice of recombining either with or without rearrangement, 
Table 7. Photoproduct distribution of benzoinoctylether as a function of the occupancy number of the micelles.

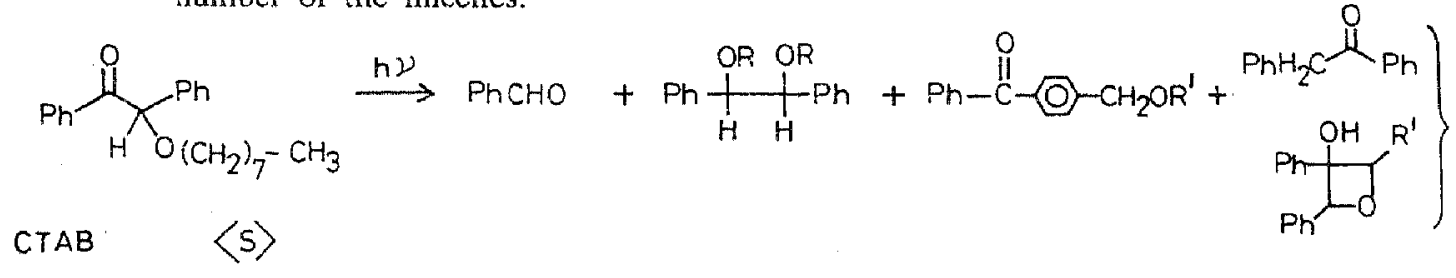

$$
\begin{aligned}
& \begin{array}{lllll}
0.5 & 26 & 12 & 47 & <5 \\
1.0 & 28 & 15 & 42 & <2 \\
2.0 & 42 & 20 & 28 & <2 \\
4.0 & 46 & 24 & 22 & <2
\end{array} \\
& \begin{array}{llllll}
\text { DTAB } & 0.5 & 63 & 35 & - & <2
\end{array} \\
& \begin{array}{lllll}
4.0 & 65 & 32 & <
\end{array}
\end{aligned}
$$
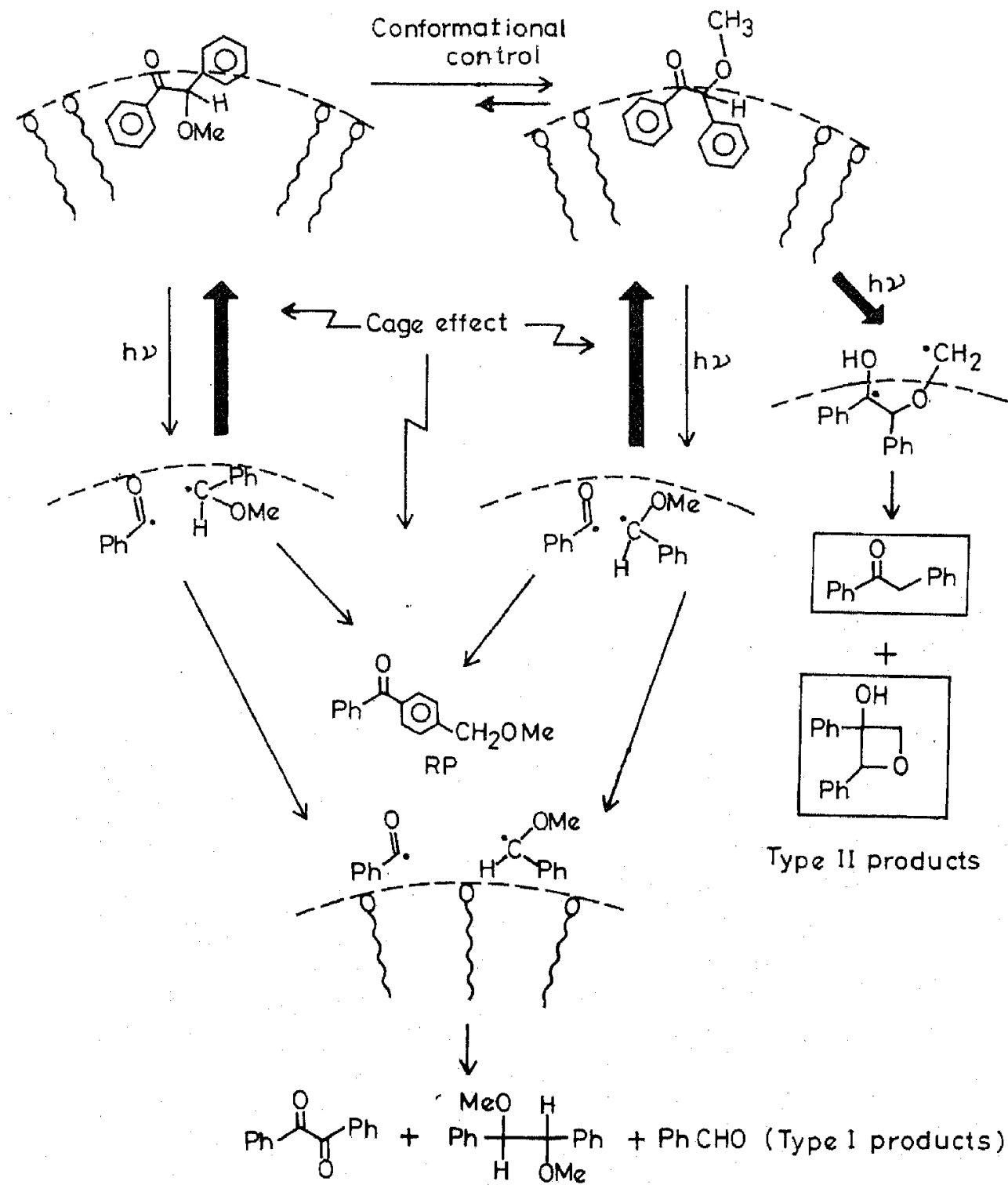

Figure 4. Conformational preference for the short chain benzoinalkylethers at the micellar interface. 


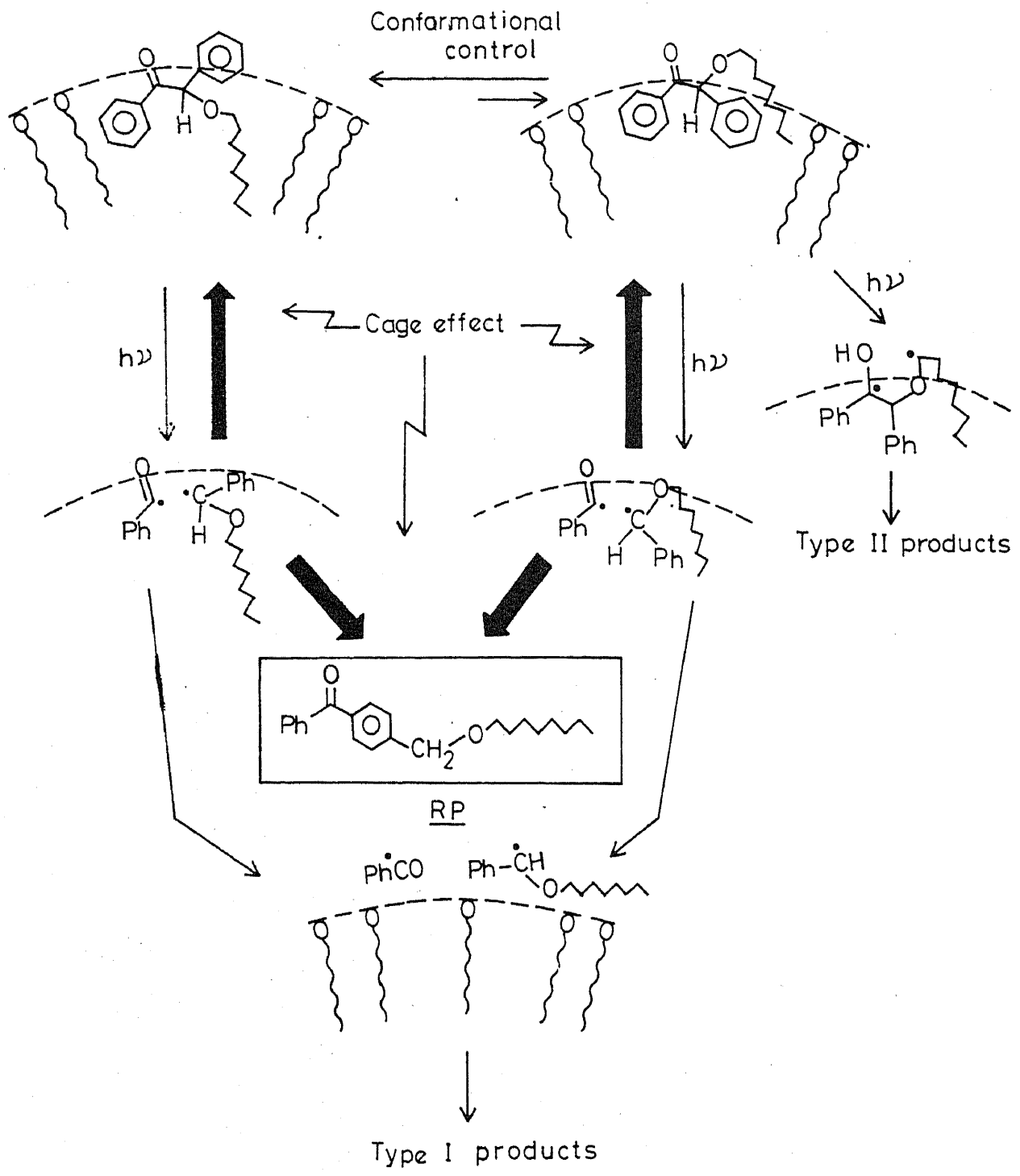

Figure 5. Conformational preference for the long chain benzoinalkylethers at the micellar interface.

thus generating benzophenones as the only products apart from the starting material itself (figure 5). The results of the present investigation reveal that excellent conformational control can be.achieved in the micellar phase by a suitable tailoring of the substrates.

In conclusion it may be stated that the increased utilisation of aqueous and micellar phases for photoreactions may prove to be of much more interest and wider scope than the use of organic solvents themselves. Much remains to be understood in the field of chemistry in organized media.

\section{References}

Abraham M H 1982 J. Am. Chem. Soc. 1042085

Ben-Naim A 1980 Hydrophobic interactions (New York: Plenum Press)

Berenjian N, de Mayo P, Sturgeon M, Sydnes L K and Weedon A C 1982 Can. J. Chem. 60426 
Breslow R, Maitra U and Rideout D C 1983 Tetrahedron Letts. 241901

Frank H S and Evans M W $1945 \mathrm{~J}$. Chem. Phys. 13507

Grieco P A, Garner P and Zhen-min He 1983a Tetrahedron Letts. 241897

Grieco P A, Garner P, Yoshida K and Huffman J C 1983b Tetrahedron Letts. 243807

Grieco P A, Yoshida K and Garner P 1983c J. Org. Chem. 483139

Kleopfer R and Morrison H 1972 J. Am. Chem. Soc. 94255

Lee K H and de Mayo P 1979 J. Chem. Soc., Chem. Commun. 493

Muthuramu K and Ramamurthy V 1982 J. Org. Chem. 473976

Muthuramu K and Ramamurthy V 1984 Indian J. Chem. B25 502

Muthuramu K, Ramnath N and Ramamurthy V 1983 J. Org. Chem. 481872

Otten J G, Yeh C S, Byrn S and Morrison H 1977 J. Am. Chem. Soc. 996353

Ramesh V and Ramamurthy V 1984a J. Org. Chem. 49536

Ramesh V and Ramamurthy V 1984b J. Photochem. 24395

Ramnath N and Ramamurthy V 1984 J. Org. Chem. 492827

Ramnath N, Ramesh V and Ramamurthy V 1985 J. Photochem. 3173

Ramamurthy V 1986 Tetrahedron (in press)

Rideout D C and Breslow R $1980 \mathrm{~J}$. Am. Chem. Soc. 1027816

Syamala M S and Ramamurthy V $1986 \mathrm{~J}$. Org. Chem. (in press)

Turro N J, Gratzel M and Braun A M 1980 Angew. Chem., Int. Ed. Engl. 19675

von Hippel P H and Schleich T 1969 Acc. Chem. Res. 2257

Wertz D H $1980 \mathrm{~J}$. Am. Chem. Soc. 1025316 